\title{
On the evaluation of the performance of asphaltene dispersants
}

\author{
Ariana A. Melendez-Alvarez ${ }^{a}$, Miguel Garcia-Bermudes ${ }^{a}$, Mohammad Tavakkoli ${ }^{a}$, Rocio H. Doherty ${ }^{a}$, \\ Siqi Meng ${ }^{\mathrm{a}}$, Dalia S. Abdallah ${ }^{\mathrm{b}}$, Francisco M. Vargas ${ }^{\mathrm{a}, *}$ \\ ${ }^{a}$ Department of Chemical and Biomolecular Engineering, Rice University, Houston, TX 77005, United States \\ ${ }^{\mathrm{b}}$ Abu Dhabi Company for Onshore Petroleum Operations Ltd., Abu Dhabi, United Arab Emirates
}

\section{A R T I C L E I N F O}

\section{Article history:}

Received 2 November 2015

Received in revised form 12 March 2016

Accepted 14 March 2016

Available online 21 March 2016

\section{Keywords}

Asphaltene inhibitor

Indirect method

Asphaltene dispersion

ADT

Dispersant assessment

\begin{abstract}
A B S T R A C T
Asphaltene precipitation and subsequent deposition is a potential flow assurance problem for the oil industry nowadays. Moreover, because oil production is moving to more difficult production environments - e.g. deeper waters - or is focusing on extracting residual oil using enhanced oil recovery techniques, the significant changes of pressure, temperature and/or composition can aggravate the asphaltene deposition problems. One of the most common strategies to prevent or at least reduce asphaltene deposition is the utilization of chemical additives. However, there are still several unresolved challenges associated to the utilization of these chemicals: First, the experimental conditions and results obtained in the lab are not always consistent with the field observations. Also, in some cases these chemical additives seem to worsen the deposition problem in the field. Therefore, there is a clear need to revisit the commercial techniques that are used to test the performance of asphaltene inhibitors and to provide a better interpretation of the results obtained. In this work, a technique based on NIR spectroscopy is presented to evaluate the performance of three commercial asphaltene dispersants. The method presented in this work is faster and more reproducible compared to the available methods such as the Asphaltene Dispersion Test (ADT) and the Solid Detection System (SDS). Also, unlike the ADT test, our proposed method can evaluate the performance of the dispersants in a wide range of temperatures and compositions. The experimental evidence shows that the asphaltene dispersants neither shift the actual onset of asphaltene precipitation nor reduce the amount of asphaltene precipitated. We believe that some results that have been reported that suggest that asphaltene dispersants can actually shift the onset of asphaltene precipitation are an unfortunate combination of insufficient sensitivity of the commercial instruments used and the slowing down of the asphaltene aggregation process by the effect of the added dispersants. The chemical additive dosage, aging time and temperature effect on the asphaltene aggregation process are also discussed in this manuscript.

With this work we aim to contribute to a better understanding of the variables that affect the performance of asphaltene dispersants, and the effect that these chemicals have on the complex multi-step mechanism of asphaltene precipitation and aggregation.
\end{abstract}

(c) 2016 Published by Elsevier Ltd.

\section{Introduction}

Asphaltenes are defined as a poly-disperse distribution of the heaviest and most polarizable fraction of the crude oil. Asphaltene characterization [1-4], chemical structure and physicochemical properties are still under investigation [5-8]. Precipitation and deposition of asphaltenes are phenomena induced by changes in pressure, temperature and composition [9,10]. During pressure depletion at low enough pressures, the asphaltene precipitation onset is reached and asphaltenes begin to precipitate [9]. At these conditions the oil expands and becomes a poor asphaltene solvent.

\footnotetext{
* Corresponding author.
}

Once asphaltenes precipitate, a multistep process begins, which involves aggregation, diffusion, advection and finally deposition of the asphaltene aggregates [9]. Oil production from deeper waters [11] and enhance oil recovery operations such as carbon dioxide injection [12], tend to worsen asphaltene deposition. Potential solutions for asphaltene deposition include physical deposits removal [10], solvent washes $[10,13]$ and chemical treatment with appropriate additives [14-16].

Asphaltene deposition in reservoirs, wells, and facilities severely impacts the oil production economics [17]. It can cause formation damage and wellbore plugging, which require expensive treatment and clean-up procedures [14], even in some extreme cases a wellbore can be completely plugged [9]. For the Gulf of 
Mexico oil fields the economic impact associated with this problematic has been estimated in USD \$70 M per well (wet tree) when well shut in for ring interventions is required [18]. If the deposition occurs in the surface controlled subsurface safety valve (SCSSV) the cost increases to USD \$100 M per well. Downtime losses based on a 10,000 BBL per day production and oil price of USD \$60 per barrel, can reach up to USD $\$ 600,000$ a day. Replacing a lost well with a side track raises the cost to around USD \$150 M [18]. Chemical additive injection for typical Gulf of Mexico production of 10,000 $\mathrm{BBL} /$ day, represents costs estimated in a range between USD $\$ 330,000$ and USD $\$ 390,000$ per well per year [18]. For Middle Eastern fields chemical additive injection represents costs in a range between USD $\$ 31,000$ and USD $\$ 46,000$ per well per year [19].

The cost of installing and maintaining asphaltene mitigation equipment and chemicals is in the millions of dollars per year [11]. Differentiation between cases with substantially impaired production requiring well intervention from those with minimal or no wellbore deposition could save the expense of installing unnecessary equipment and injecting chemical inhibitors when they are not needed as well as helping in the development of improved chemicals for prevention of wellbore deposition problems [11].

Extensive research has been already conducted to elucidate the variables that affect additives performance [15,17,20-23]. It has been reported that asphaltene characteristics [21,24,25], solvent condition [15,23,26,27], additive structure [20,21,27], and additive amount adsorbed on asphaltenes $[2,23,26]$ influence additive performance. In the case of dispersant additives it is accepted that dispersant interaction with asphaltenes decreases the aggregates size of precipitated asphaltene and/or hinders possible subsequent aggregation [28]. However, in other cases the dispersants seem to promote the aggregation [23]. The study of asphaltene dispersant additives has shown that additives can have different performance, acting as stabilizers, enhance aggregation or they can have no effect [14]. Chemical additives efficiency is typically measured in terms of a delay in the detection of asphaltene precipitation $[15,29,30]$. At this point it is important to alert the reader that asphaltene precipitation is a necessary but not sufficient condition for asphaltene deposition [9,31].

Some methodologies such as microscopy [10,32], polarized light microscopy [33], solid detection system [15], filtration [22], filter drop spreading method [29,34] and spectroscopy [35] have been used to evaluate the performance of chemicals intended to reduce or prevent asphaltene deposition problems. One of the most common and widely used commercial techniques is the Asphaltene Dispersion Test (ADT) [12]. To evaluate the tendency of asphaltenes to precipitate and the efficiency of chemical additives, the ADT uses normal gravimetric sedimentation measured over a period of time after diluting the oil with an asphaltene precipitant such as n-heptane [12].

The Solid Detection System (SDS) is another technique that is used by service laboratories to evaluate the stability of asphaltenes. Unlike the ADT test, the SDS can analyze live oil samples at high pressure and high temperature (HPHT) to determine the asphaltene onset pressure (AOP) with and without the addition of asphaltene dispersants.

In this work, a technique based on NIR spectroscopy is presented to evaluate the performance of three commercial asphaltene dispersants at ambient pressure. This technique is faster and more reproducible compared to the available tests such as the Asphaltene Dispersion Test (ADT) and the Solid Detection System (SDS). Furthermore, we have been able to reproduce the effect of inhibitors on the shift of the onset of asphaltene precipitation that is obtained by the Solid Detection System (SDS) at high pressure and temperature, using dead-oil samples at ambient pressure.
The experimental evidence shows that the asphaltene dispersants neither shift the actual onset of asphaltene precipitation nor reduce the amount of precipitated asphaltene. We believe that the results obtained by the SDS that suggest that asphaltene dispersants can actually shift the onset of asphaltene precipitation are an unfortunate combination of insufficient sensitivity of the instrument and the slowing down of the asphaltene aggregation process by the effect of the added dispersants. The chemical additive dosage, aging time and temperature effect on the asphaltene aggregation process are also studied in this work.

\section{Experimental section}

\subsection{Materials}

Experiments were conducted with crude oil S and crude oil A from the Middle East. The crude oil S was decanted and centrifuged to remove aqueous phase and suspended particles. The properties for this crude oil have been reported previously [36], and are listed in Table 1 along with the properties of the crude Oil A. The commercial asphaltene dispersants 8,9 and 15 were used to treat the crude oil samples. All the reagents used for the experiments were a high performance liquid chromatography (HPLC)-grade and were procured from Sigma-Aldrich.

\subsection{Asphaltene dispersant test (ADT)}

The crude oil S was treated with dispersants 8,9 and 15 . Different chemical dosages were tested. For dispersant 8, 9 and 15, 70 and $500 \mathrm{ppm}$ were prepared and analyzed. All the solutions were prepared by diluting a concentrated solution, e.g. $3000 \mathrm{ppm}$, of the corresponding dispersant in crude oil. The homogenization of the sample was achieved in a closed beaker for a period of one hour using a magnetic stirrer at $700 \mathrm{rpm} .250 \mu \mathrm{L}$ of the corresponding crude oil sample were placed in graduated centrifuge tubes and mixed with $9.75 \mathrm{~mL}$ of $\mathrm{n}$-heptane. A sample of crude oil with no dispersant was used as a control.

Finally, the samples were left undisturbed for a specific period of time (also known as aging time) and the amount of sediment obtained was recorded in $\mathrm{mL}$ at the end of the experiment. The aging times used were $1 \mathrm{~h}, 24 \mathrm{~h}$ and 1 week. When no sediment was observed the data entry was recorded as "clear" and when precipitation was observed but it was not measurable the data entry was recorded as "trace" [12]. The criterion for evaluation of the performance of dispersants using the Asphaltene Dispersion Test (ADT) consists in comparing the amount of sediment obtained in $\mathrm{mL}$ for the different chemicals and dosages with respect to the control or untreated oil, which was the mixture of crude oil and nheptane without the addition of the dispersant.

\subsection{Solid detection system}

This instrument measures the transmittance of light at a fixed wavelength in the near infrared (NIR) region it is usually combined

Table 1

Crude oil A properties at $15^{\circ} \mathrm{C}$ and $1 \mathrm{~atm}$ and crude oil S properties at $20^{\circ} \mathrm{C}$ and $1 \mathrm{~atm}$.

\begin{tabular}{lll}
\hline Property & Crude oil S value & Crude oil A value \\
\hline Density $\left(\mathrm{g} / \mathrm{cm}^{3}\right)$ & 0.843 & 0.763 \\
Molecular weight $(\mathrm{g} / \mathrm{mol})$ & 193 & 177 \\
Viscosity $(\mathrm{cP})$ & 9.5 & 1.057 \\
Saturate $(\mathrm{wt} \%)$ & 66.26 & 68.9 \\
Aromatic $(\mathrm{wt} \%)$ & 25.59 & 21.8 \\
Resin $(\mathrm{wt} \%)$ & 5.35 & 7.1 \\
$\mathrm{n}-\mathrm{C}_{5}$ Asphaltene (wt\%) & 2.8 & 0.5 \\
\hline
\end{tabular}


with high pressure-microscopy (HPM) images to determine the asphaltene onset pressure (AOP) at a particular temperature and composition. The crude oil sample is loaded into the SDS cell and it is stabilized at the temperature and pressure of interest. Then, the fluid is depressurized step-wise. During depressurization, the light transmittance that passes through the sample is measured and images using the HPM are recorded.

\subsection{Direct method for detection of asphaltene precipitation}

The blends of crude oil and precipitant, with and without dispersants, were prepared following the same procedure that was explained in Section 2.2. Blends of different ratios of crude oil (with and without dispersant) and n-heptane were prepared ranging from 0 to 90 vol.\% of $n$-heptane. The test tubes were shaken by hand and the blends were transferred to $10-\mathrm{mm}$ path length quartz cuvettes, which were then tightly closed. To maintain a homogeneous temperature the samples were kept inside a Jeio Tech oven model OV-11/12 at a specific temperature. $25^{\circ} \mathrm{C}$ and $70^{\circ} \mathrm{C}$ were used in this work. The samples were allowed to rest undisturbed for the specified aging time. In this case the aging time is the period of time allotted between sample preparation and the measurement of the NIR transmittance. The masses of added oil and precipitant were measured and the actual volumes were back calculated assuming an ideal solution. Then, the NIR transmittance was measured using a Shimadzu UV-Vis-NIR spectrophotometer Model UV-3600 equipped with a "TC1 Temperature Controller" and a "t $2 \times 2$ Dual Temperature-Controlled Cuvette Holder" provided by Quantum Northwest. Inside this chamber the samples were mixed at $1000 \mathrm{rpm}$ with a magnetic stirrer that was placed inside the cuvette. During the measurement the samples were stirred to prevent the sedimentation of any asphaltene aggregates that could be present in the system. This technique provided a more reproducible result, compared to static unstirred systems in which the sedimentation of the asphaltene aggregates can affect the results.

The transmittance values were measured for wavelengths ranging between 1000 and $1300 \mathrm{~nm}$. It was found that different wavelengths in this range can be used to detect the onset of precipitation as long as the signal is not saturated. At wavelengths below $1000 \mathrm{~nm}$, it was not possible to obtain a measurement due to the signal saturation. The relative masses of oil and precipitant present in each blend were measured and the corresponding volumes were back-calculated using the density of the components, assuming an ideal mixing. The transmittance values of the different samples were corrected by subtracting the transmittance of n-heptane and then the effect of dilution was mathematically removed. The details for the correction of the dilution effect have been explained by Tavakkoli et al. [31]. The corrected transmittance values were then normalized using the transmittance of the crude oil as the reference. The normalized values are usually plotted as a function of the volume fraction of n-heptane or crude oil. However, the same results can also be presented in terms of light intensity (i.e. light transmittance) instead of absorbance, which is how the results are usually reported by direct spectroscopy. In the latter case, asphaltene precipitation was determined by the decrease in the normalized light intensity caused by asphaltene aggregates that block the path of light. The first deviation from the linear trend indicated the precipitation of asphaltenes. The normalized light intensity can be plotted as a function of the volume fraction of crude oil and/or n-heptane.

After the NIR transmittance values were recorded, the samples were observed under a microscope AmScope model B340, coupled with a MU300 microscope digital camera operating at a combined magnification of $400 \times$ to confirm the existence of asphaltene aggregates.

\subsection{Indirect method for detection of asphaltene precipitation}

Because precipitation is measured independently of aggregation using the indirect method [31], the chemical additive effect on asphaltene precipitation and aggregation phenomena can be independently studied. The indirect method is a combination of gravimetric and spectroscopic techniques and the details of this technique can be found in the work of Tavakkoli et al. [31].

The term "indirect" refers to the quantification of asphaltenes that remain in solution, after precipitation and centrifugation of the samples. The underlining principle of this method is that submicron particles that are not detected by NIR light scattering techniques and optical microscopy can be settled down by high speed centrifugation. The removal of these particles affects the transmittance of the supernatant liquid, which, in turn, can be used to detect the point of asphaltene precipitation. The behavior observed with this methodology follows Beer-Lambert law which states that the absorbance is a linear function of the solution concentration [31].

The crude oil S was treated with dispersants 8 and 9. Different chemical dosages were tested. For dispersant 8 , dosages of 70 and $500 \mathrm{ppm}$ were used, whereas for dispersant 9, samples containing 70,500 and $2000 \mathrm{ppm}$ were prepared and analyzed. The crude oil and all the solutions were prepared as explained in Section 2.2.

Blends of different ratios of crude oil (with and without dispersant) and n-heptane were prepared as explained in Section 2.4. In this case the aging time is the period of time allotted between sample preparation and centrifugation, prior to the spectroscopic measurements. The measured values were corrected to account for the effect of dilution and then normalized using the absorbance of the crude oil sample, in the same way it was explained in Section 2.4.

\section{Results and discussion}

Commercial chemical additives have been tested to study their effect on asphaltene precipitation and aggregation phenomena using two different techniques: "Indirect method" and "Direct spectroscopy", which have been explained in Section 2.

Fig. 1(a) presents the HPHT test results for crude oil A and Fig. 1 (b) shows direct spectroscopy results for crude oil $\mathrm{S}$ at ambient conditions. Crude oils S and A were obtained from two different wells from the same oil field. To induce the precipitation of asphaltenes at ambient conditions, n-heptane was added to crude oil $\mathrm{S}$, which gives the driving force for asphaltene precipitation, instead of the pressure depletion that is used in the HPHT experiments. Fig. 1(b) presents the light intensity vs. crude oil and heptane vol.\%. According to this figure the light intensity increases as the crude oil is diluted by the addition of the precipitant, which is the same trend that is observed in the case of HPHT experiments. In the latter case the dilution of the sample is caused by the expansion of the fluid. In either case, when the driving force for precipitation is high enough, the formation of asphaltene aggregates causes a drop on transmittance of light. In the case of the experiment performed at ambient pressure, according to Fig. 1(b), the addition of $n$-heptane first dilutes the asphaltenes in solution (0-40\% n-heptane), then it causes their precipitation (40-60\% n-heptane) and finally, as more n-heptane is added, both the asphaltene aggregates and the components from the oil that remain in solution are diluted, increasing the transmittance of the light (>60\% n-heptane). From Fig. 1(a) and (b) it is clear that results from direct spectroscopy performed at ambient conditions and the results obtained at high pressure and temperature show similar trends before and at the onset of asphaltene precipitation. However, unlike the experiments conducted at ambient pressure, in which the effect of dilution is observed after the onset of 

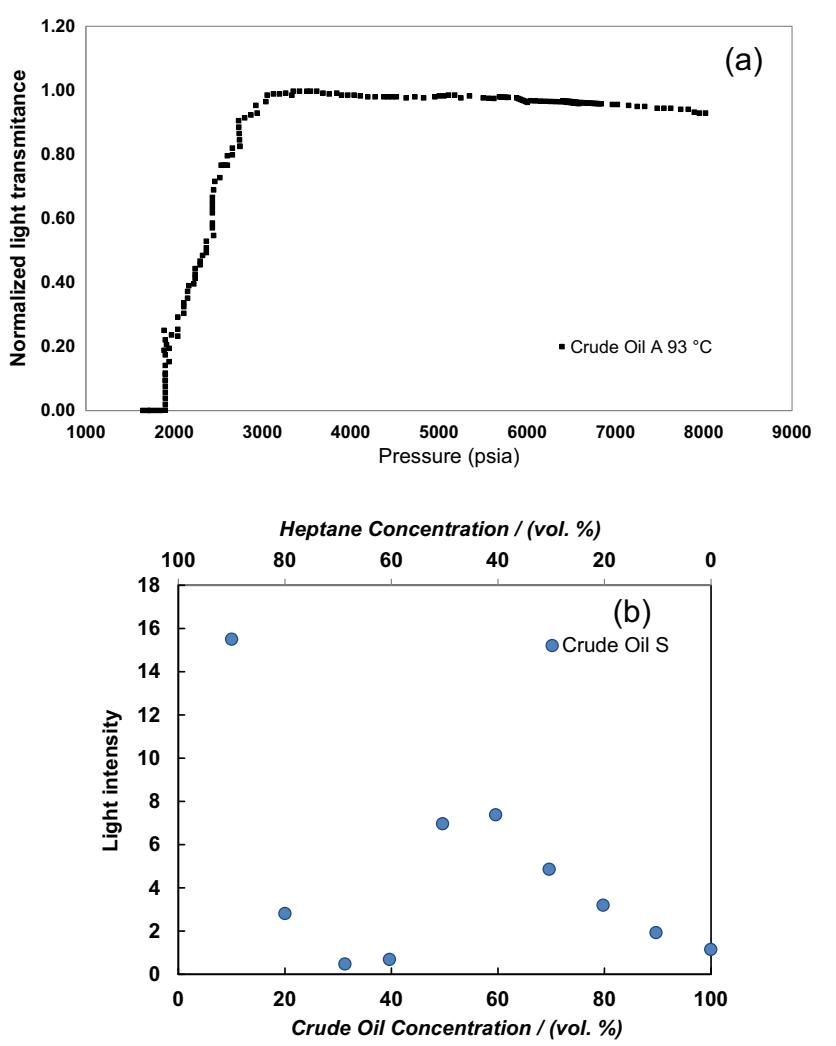

Fig. 1. Results for crude oil A and crude oil S, (a) results for AOP measurement using SDS for crude oil A at $93{ }^{\circ} \mathrm{C}$, (b) direct spectroscopy results for crude oil S diluted with n-heptane and aged for $1 \mathrm{~h}$ at $25^{\circ} \mathrm{C}$.

precipitation, in the case of the high pressure and temperature experiments, at low enough pressure the bubble point is attained and the composition of the remaining liquid is no longer constant. In fact, as the pressure decreases below the bubble point the concentration of asphaltene increases in the liquid phase, which in turn reduces the transmittance of light. Moreover, due to the formation of the gas bubbles the transmittance that is recorded below the bubble point pressure does not correspond to that of the liquid phase.

The results of the experiments at ambient pressure can be presented in a different way, by: (1) removing the effect of dilution, (2) normalizing the light intensity data and (3) plotting the results in a logarithmic scale. Fig. 2 presents the same experimental results as Fig. 1(b), after the implementation of the three transformation steps. The logarithmic scale is needed to enhance the changes of the light intensity for high concentrations of nheptane. Also, with this post-processing technique, the onset of asphaltene precipitation can be readily obtained as the point where the first deviation from the horizontal line is observed. Therefore, this technique to process and report the experimental data will be used in the rest of the manuscript for direct spectroscopy results and its interpretation, which will be discussed in detail in Section 3.3. The average of repeated experiments is plotted in the figures and the Average Standard Deviation (ASD) is reported in the figure caption.

\subsection{Asphaltene dispersion test}

The crude oil S was treated with dispersants 8,9 and 15 at 70 and $500 \mathrm{ppm}$ as described in Section 2.1. The results are shown in Fig. 3 and the volume of sediment obtained in the different cases is presented in Table 2. The ADT ranks the efficiency of the

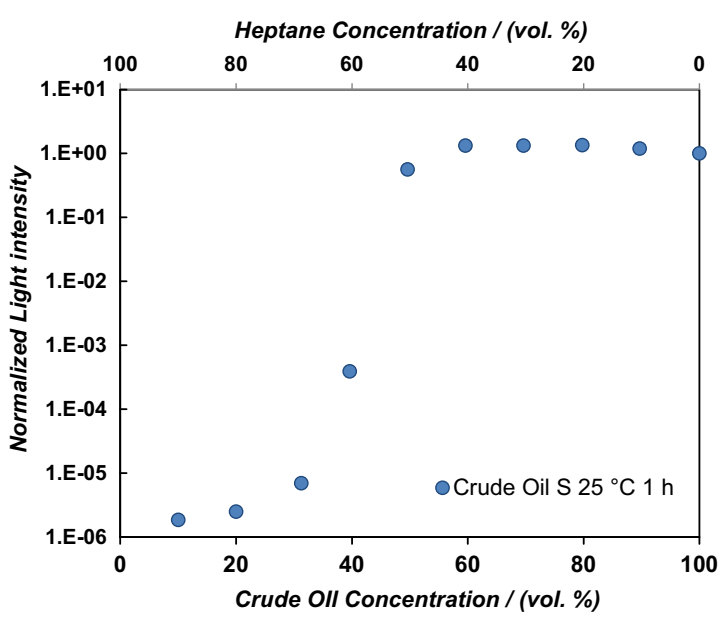

Fig. 2. Direct spectroscopy results for crude oil S diluted with n-heptane and aged for $1 \mathrm{~h}$ at $25^{\circ} \mathrm{C}$. Light intensity has been normalized and corrected to remove the effect of dilution. The ASD is $6.03 \%$.

chemical additives based on their ability to disperse the asphaltene aggregates, which in turn reduces the terminal velocity of these particles and, consequently, the amount of sediment obtained [12]. The lower the amount of sediment collected the more efficient the dispersion of asphaltenes.

After $24 \mathrm{~h}$ only the samples treated with dispersant 8 and 15 at $500 \mathrm{ppm}$ did not show any sediment. After $1 \mathrm{~h}$ the untreated crude oil presented sediments and at longer times the sediments level decreased as reported in Table 2. Further analysis, which will be reported in Section 3.3, showed that the amount of precipitated asphaltene actually increases with longer aging times. Therefore the reduction of the level of sediment could be the result of sediment compaction over time. The same observation is applicable to dispersant 9 and 15 at $70 \mathrm{ppm}$.

In addition, the ADT test requires large amounts of heptane to obtain a clear sample that allows sediment observation through it (97\% heptane or more), the result obtained from ADT could be misleading because the dispersant cannot be tested at the heptane concentration that causes the highest precipitation of asphaltene and/or the largest asphaltene aggregates. Also, this high dilution may not be representative of the conditions at which asphaltenes precipitate and aggregate in real systems.

\subsection{Solid detection systems}

Fig. 4 presents the SDS results obtained for crude oil A without the addition of dispersant. According to this figure the light transmittance increases as the pressure decreases from 8000 to 3500 psi. At a pressure below 3500 psi the light transmittance starts to decrease. $3500 \mathrm{psi}$ is the AOP. The reported uncertainty for this measurement is about 200 psi. Fig. 4 also shows the result for crude oil A treated with $100 \mathrm{ppm}$ of commercial dispersant 9. In this case the AOP drops to $2500 \mathrm{psi} \pm 200 \mathrm{psi}$. The AOP shift can be clearly observed in Fig. 4. At this point, it is important to alert the reader that the shift on the recorded AOP upon addition of the dispersant does not mean that the thermodynamic stability of asphaltene was actually affected. This point will be further discussed in Section 3.4.

\subsection{Effectiveness of commercial asphaltene dispersants}

Fig. 5 presents the results for the crude oil S with and without dispersant 8 at $70 \mathrm{ppm}$. The crude oil without dispersant 


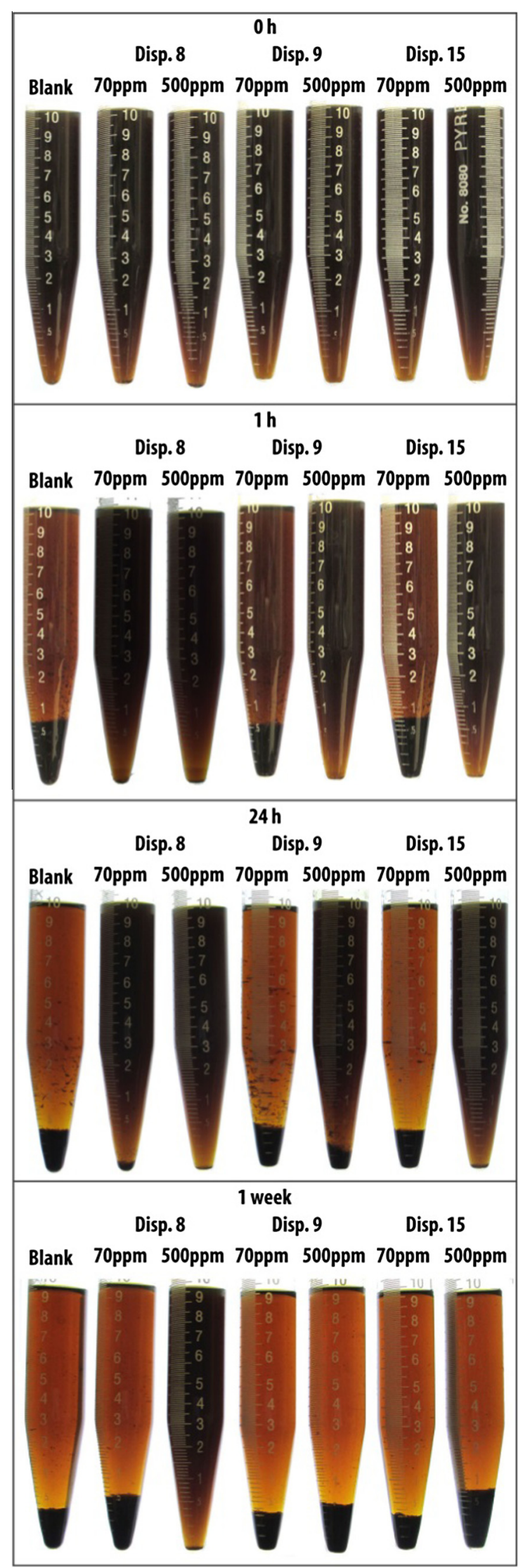

Fig. 3. ADT results for crude oil $S$ treated with dispersants 8,9 and 15 at $70 \mathrm{ppm}$ and $500 \mathrm{ppm}$ for aging times of $0 \mathrm{~h}, 1 \mathrm{~h}, 24 \mathrm{~h}$ and 1 week.
Table 2

ADT results for crude oil S treated with dispersants 8, 9 and 15 at 70 and $500 \mathrm{ppm}$.

\begin{tabular}{lcllll}
\hline Sample & Dosage $(\mathrm{ppm})$ & $0 \mathrm{~h}(\mathrm{ml})$ & $1 \mathrm{~h}(\mathrm{ml})$ & $24 \mathrm{~h}(\mathrm{ml})$ & 6 days $(\mathrm{ml})$ \\
\hline Crude oil S & 0 & Clear & 0.7 & 0.4 & 0.3 \\
With disp 8 & 70 & Clear & Clear & Trace & 0.5 \\
& 500 & Clear & Clear & Clear & Clear \\
With disp 9 & 70 & Clear & 0.5 & 0.4 & 0.3 \\
& 500 & Clear & Clear & 0.2 & 0.5 \\
With disp 15 & 70 & Clear & 0.7 & 0.4 & 0.3 \\
& 500 & Clear & Clear & Clear & 0.7 \\
\hline
\end{tabular}

corresponds to the control. Fig. 5 has two sections: the first section is a nearly horizontal line at low precipitant amounts and high crude oil vol.\%. In this section both systems present a similar response. Then, there is a second section at high precipitant amounts and low crude oil vol.\% where the light intensity values deviate from the horizontal trend. The point at which this deviation occurs represents the volume fraction of precipitant required for the detection of asphaltene precipitation.

In the second section after the detection of asphaltene precipitation, Fig. 5 shows a progressive light intensity reduction for the control. The light intensity decrease indicates an increase in the number and size of asphaltene aggregates. For the system treated with dispersant 8 the light intensity dropped at a slower rate than the control indicating a slower precipitation and aggregation rates under the effect of dispersant 8 . Then, when the heptane concentration approached 100 vol.\% the treated system showed a second inflection point at $70 \mathrm{vol} . \%$ where the light intensity increased again approaching the horizontal base line that represents a stable solution indicating the presence of less and/or smaller aggregates. Addition of n-heptane has two effects on the precipitation of asphaltenes: on one hand it dilutes the asphaltene content and therefore its light intensity decreases, even when there is no precipitation (at volume fractions below 50 vol.\%), on the other hand it slows down the precipitation and aggregation kinetics. The first effect can be accounted for by multiplying the measured transmittance by the dilution factor. In the presence of a dispersant the kinetics of asphaltene precipitation and aggregation is further reduced and therefore the light intensity is greater than the crude oil $\mathrm{S}$, for a given aging time. As the aging time increases the light intensity usually drops and the difference between treated and untreated samples is reduced. This will be discussed in detail in Section 3.3.2.

\subsubsection{Effect of dosage}

To understand the effect of the dosage of the dispersants 8,9 and 15 , studies with different concentrations of each dispersant at $70 \mathrm{ppm}$ and $500 \mathrm{ppm}$ were performed, at $25^{\circ} \mathrm{C}$ and $1 \mathrm{~h}$ aging time. Lower dosages of dispersants are desirable for field applications in an attempt to minimize the treatment cost. The results are shown in Fig. 6.

As observed from Fig. 6(a) and (b) dispersant 8 had similar results at the two different dosages. The benefit was marginally better for the higher dispersant dosage and only for heptane concentrations of $70 \mathrm{vol} . \%$ and $80 \mathrm{vol} . \%$. This is in agreement with the idea that there is an optimal concentration of asphaltene dispersant and, depending on the system characteristics, the size of the asphaltene aggregates remains nearly constant even if more dispersant is added [14]. According to the ADT results discussed in Section 3.1, after $1 \mathrm{~h}$ for dispersant 8 at $500 \mathrm{ppm}$ there was not significant sedimentation. However, from the direct spectroscopy results it was observed that there was asphaltene 


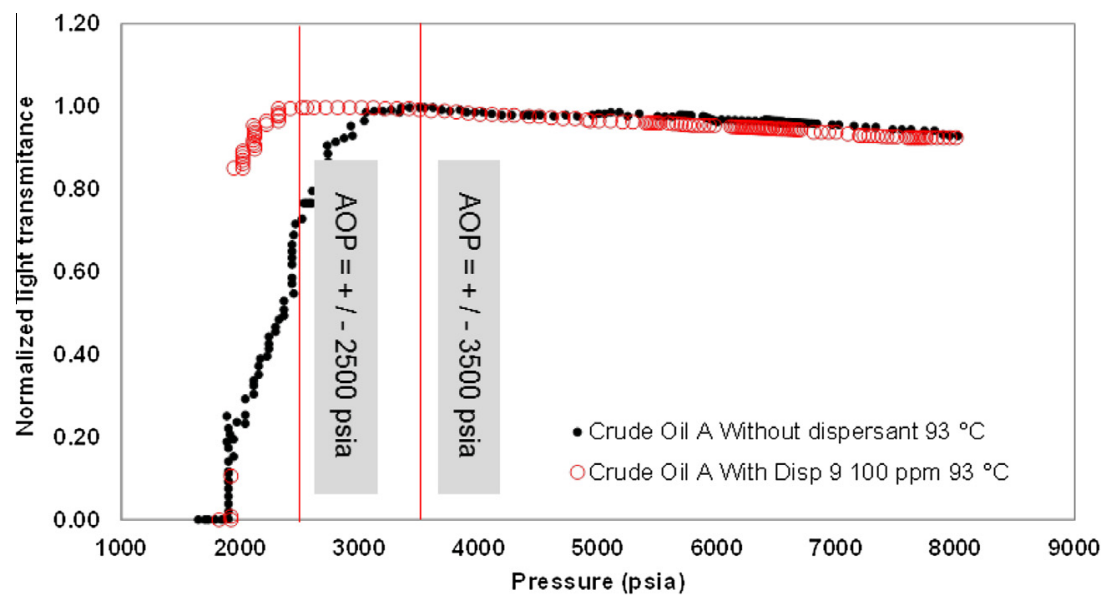

Fig. 4. Results for AOP measurement using SDS at $93{ }^{\circ} \mathrm{C}$ for crude oil A with $100 \mathrm{ppm}$ of dispersant 9 and without dispersant.

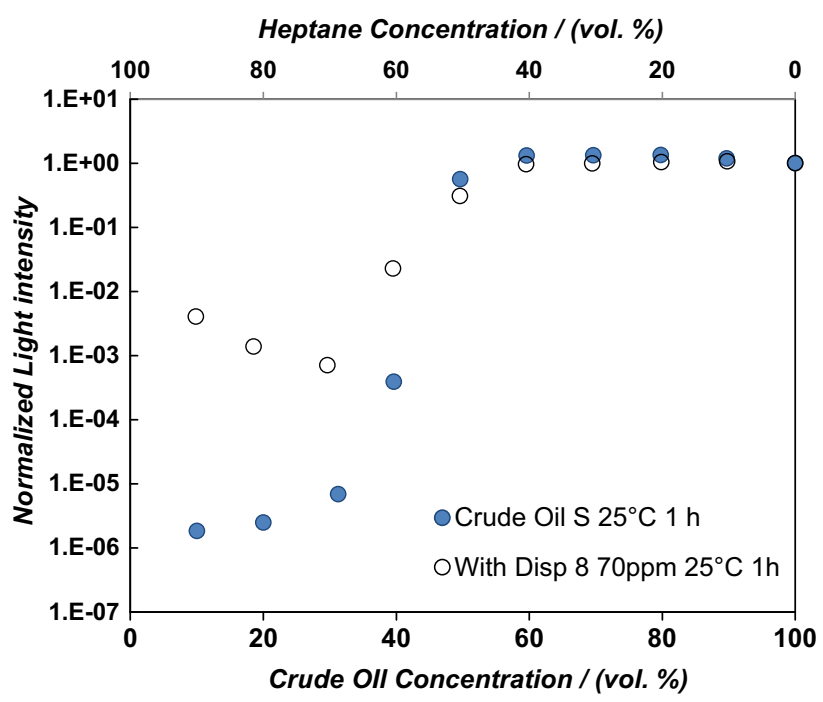

Fig. 5. Results of the direct method for the crude oil S vs. Dispersant 8 with a 70 ppm dosage, after mixing with heptane and aging for $1 \mathrm{~h}$ at $25^{\circ} \mathrm{C}$. The ASD are $6.03 \%$ and $5.31 \%$ for the crude oil S and dispersant 8 respectively.

precipitation and aggregation in the presence of dispersant 8 at $500 \mathrm{ppm}$ after one hour, but it was slower compared to the aggregation observed in the crude oil $\mathrm{S}$.

On the other hand, Fig. 6(a) and (b) showed for dispersants 9 and 15 that increasing the dispersant dosage causes a reduction on the asphaltene aggregation and consequently and increase on the light transmittance. At $500 \mathrm{ppm}$ both dispersants 9 and 15 significantly reduced asphaltene aggregation.

According to Fig. 6(b), when Crude oil S was treated with dispersants 8,9 and 15 at 500 ppm all the samples showed almost the same level of performance. However, at the lower dosage of $70 \mathrm{ppm}$, inhibitor 8 outranked inhibitors 9 and 15. Therefore, according to this technique inhibitor 8 at $70 \mathrm{ppm}$ seems a reasonable choice. Treating a well at high dosages is not economically viable as it was previously discussed in Section 1.

Table 3 shows the detection of asphaltene precipitation for treated crude oil S with $70 \mathrm{ppm}$ and $500 \mathrm{ppm}$ of dispersant after $1 \mathrm{~h}$ aging time. The detection of asphaltene precipitation was calculated fitting regression curves for the first section and the beginning of the second section of the figures and finding their intersection. Additional detail is provided in the supporting
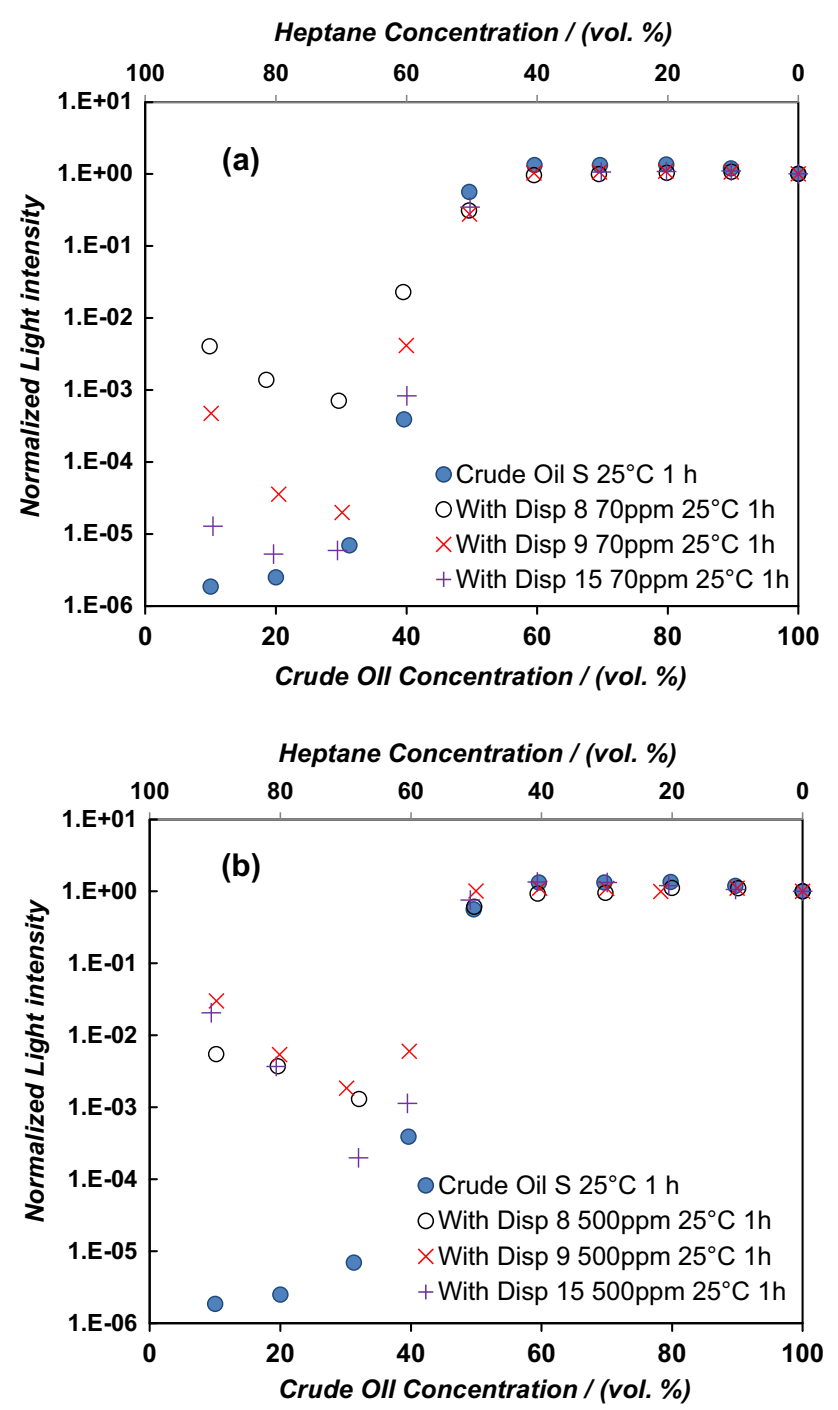

Fig. 6. Results of the direct method for the crude oil S with, (a) dispersant 8, 9 and 15 at 70 ppm, (b) dispersant 8,9 , and 15 at 500 ppm, after mixing with heptane and aging for $1 \mathrm{~h}$ at $25{ }^{\circ} \mathrm{C}$. The ASD are $6.03 \%, 5.31 \%, 6.82 \%, 3.21 \%$, for the crude oil S, dispersant 8,9 and 15 at 70 ppm, and 2.43\%, 2.62\%, 0.46\%, for dispersant 8,9 and 15 at $500 \mathrm{ppm}$, respectively. 
Table 3

Detection of asphaltene precipitation of crude oil S with 70 and 500 ppm dosage of different commercial dispersants, after mixing with heptane and aging for $1 \mathrm{~h}$ at $25^{\circ} \mathrm{C}$.

\begin{tabular}{lll}
\hline Sample & \multicolumn{2}{l}{$\begin{array}{l}\text { Detection of asphaltene precipitation } \\
\text { (n-heptane vol.\%) }\end{array}$} \\
\cline { 2 - 3 } & $70 \mathrm{ppm}$ & $500 \mathrm{ppm}$ \\
\hline Crude oil S & $48.8 \pm 2.6$ & $48.8 \pm 2.6$ \\
With dispersant 8 & $46.2 \pm 3.0$ & $49.5 \pm 1.9$ \\
With dispersant 9 & $45.5 \pm 1.9$ & $50.1 \pm 1.8$ \\
With dispersant 15 & $47.6 \pm 1.3$ & $49.8 \pm 2.1$ \\
\hline
\end{tabular}

information section. The control used for comparison is the crude oil S without inhibitor. The onset of asphaltene precipitation shows a slight change upon addition of the asphaltene dispersant.

\subsubsection{Effect of the aging time}

As it was previously defined in Section 2.3 the aging time corresponds to the time elapsed between sample preparation and the measurement of the light intensity. The effect of aging time on the detection of asphaltene precipitation and aggregation phenomena as well as on the performance of different dispersants is considered in this section. Fig. 7 shows direct method results for the control experiment and crude oil $\mathrm{S}$ treated with dispersants 8 and 9 at $70 \mathrm{ppm}$ for different aging times, namely $1 \mathrm{~h}, 24 \mathrm{~h}$ and 2 weeks. The dispersant 15 was not evaluated because it did not show a significant effect after $1 \mathrm{~h}$ at $70 \mathrm{ppm}$ as discussed in Section 3.3.1 Fig. 7 shows the results for all aging times for dispersant 8 at $70 \mathrm{ppm}$. A similar trend was observed with dispersant 9 the results are included in the supporting information section. In addition, for all the treated and untreated systems the asphaltene aggregation was a function of time and increased as time passed. Thus, with time, the asphaltenes that have precipitated and have been detected after one hour continue to aggregate even in the presence of asphaltene dispersants. This suggests that the dispersants slow the aggregation process down, but they cannot prevent the occurrence of the asphaltene precipitation or aggregation.

The detection of asphaltene precipitation was determined and the results are reported in Table 4. The volume of n-heptane needed to induce the precipitation of asphaltene significantly decreased when the aging time increased from one hour to two weeks. According to Table 4, the effect of the aging time is much

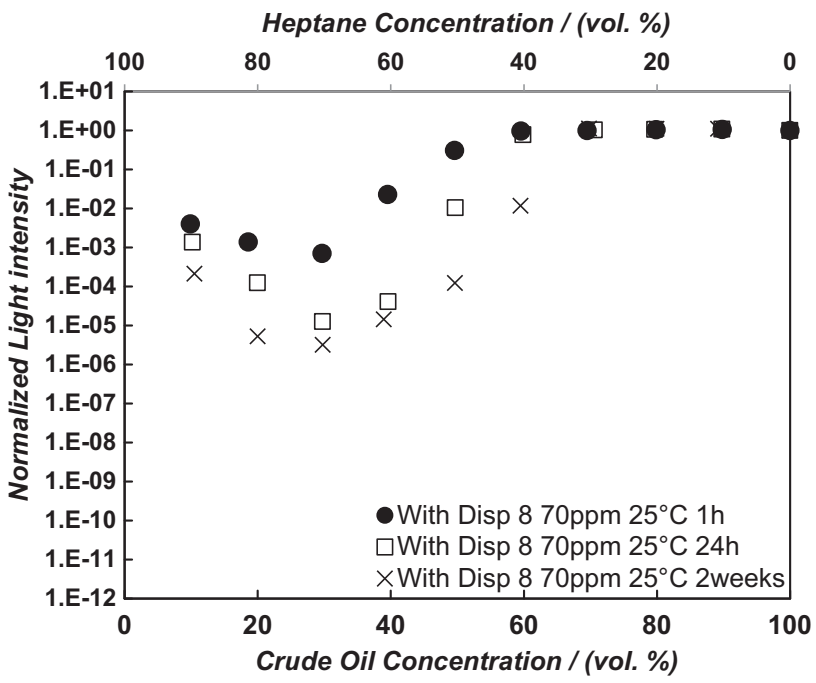

Fig. 7. Results of the direct method for the crude oil S with dispersant 8 at $70 \mathrm{ppm}$ after mixing with heptane and aging for $1 \mathrm{~h}, 24 \mathrm{~h}$ and 2 weeks at $25^{\circ} \mathrm{C}$. The ASD are $5.31 \%, 2.18 \%, 1.79 \%$ for dispersant 8 at $1 \mathrm{~h}, 24 \mathrm{~h}$ and 2 weeks, respectively.
Table 4

Detection of asphaltene precipitation of crude oil S treated with different commercial dispersants, at $70 \mathrm{ppm}$ after mixing with heptane and aging for $1 \mathrm{~h}, 24 \mathrm{~h}$ and 2 weeks at $25^{\circ} \mathrm{C}$.

\begin{tabular}{llll}
\hline Sample & \multicolumn{3}{l}{$\begin{array}{l}\text { Asphaltene Onset Concentration (AOC) (n-heptane } \\
\text { vol.\%) }\end{array}$} \\
\cline { 2 - 4 } & $1 \mathrm{~h}$ & $24 \mathrm{~h}$ & 2 weeks \\
\hline Crude oil S & $48.8 \pm 2.6$ & $42.4 \pm 1.6$ & $28.3 \pm 0.6$ \\
With disp 8 & $46.2 \pm 3.0$ & $41.3 \pm 1.3$ & $29.7 \pm 0.1$ \\
With disp 9 & $45.5 \pm 1.9$ & $39.5 \pm 0.9$ & $32.4 \pm 1.7$ \\
\hline
\end{tabular}

more prominent than the effect of the asphaltene dispersant. For both untreated and treated systems the longer the aging time the less precipitant is needed to induce the asphaltene precipitation.

\subsubsection{Effect of temperature}

In order to study the temperature effect on the performance of the different dispersants, experiments at $25^{\circ} \mathrm{C}$ and $70^{\circ} \mathrm{C}$ were conducted for $24 \mathrm{~h}$. The same dosages used for the dispersant effect in Section 3.3.1 were evaluated.

It is important to consider the effect of temperature on the thermodynamic properties of the system. Temperature variations induce changes in asphaltene solubility, crude oil density and viscosity, since all these properties are temperature dependent. The solubility of asphaltenes in oil usually increases with increasing temperature, because at this phase boundary temperature, the entropy gain from mixing just overcomes the enthalpically favored phase splitting [37]. Moreover, due the polydisperse nature of the asphaltenes, the asphaltene fraction that precipitates at high temperature may differ from that precipitates at lower temperature. Different asphaltene fractions have different properties such as density and diffusion rate. Only the more unstable asphaltenes precipitate at higher temperatures. Thus, the effect of temperature on the performance of a given dispersant can be very significant.

Fig. 8 shows the effect of temperature on blends of oil and nheptane with and without dispersants. The system appeared to be more stable at $70{ }^{\circ} \mathrm{C}$ than at $25^{\circ} \mathrm{C}$ without the presence of the dispersant. From the results for the crude oil or control experiment it is observed that the aggregation and precipitation rates were lower when the temperature increased. At higher temperature, the crude oil was a better solvent for the asphaltenes and more precipitant was needed to induce their precipitation. Fig. 8(a) and (b) shows the results for dispersant 8 at $70 \mathrm{ppm}$ and $500 \mathrm{ppm}$ at $25^{\circ} \mathrm{C}$ and $70^{\circ} \mathrm{C}$ after $24 \mathrm{~h}$ aging time, respectively. It can be observed for dispersant 8 at 70 ppm that the difference with respect to the control experiment was higher at $25^{\circ} \mathrm{C}$ than at $70^{\circ} \mathrm{C}$. In other words, at $25^{\circ} \mathrm{C}$ dispersant 8 reduced the aggregation more effectively than at $70^{\circ} \mathrm{C}$. Therefore, when the temperature increased the dispersant performance decreased.

Analogous experiments were conducted with dispersant 9 at $70 \mathrm{ppm}, 25^{\circ} \mathrm{C}$ and $70{ }^{\circ} \mathrm{C}$ for $24 \mathrm{~h}$. At $25^{\circ} \mathrm{C}$ dispersant 9 did not disperse the asphaltene aggregates as effectively as dispersant 8 but it had some effect. At $70{ }^{\circ} \mathrm{C}$ dispersant 9 performance decreased and no dispersion effect was observed. The results are included in the supporting information. Dispersant 15 was also studied at $70 \mathrm{ppm}$ and $70^{\circ} \mathrm{C}$ and the results showed poor performance. As was previously discussed in Sections 3.3.1 and 3.3.2 dispersant 15 was not able to disperse the asphaltenes even at room temperature condition.

\subsubsection{Definition of dispersive performance}

To facilitate the ranking of the different asphaltene dispersants, a dispersive performance efficiency (DPE) is proposed. The efficiency is calculated as follows: 

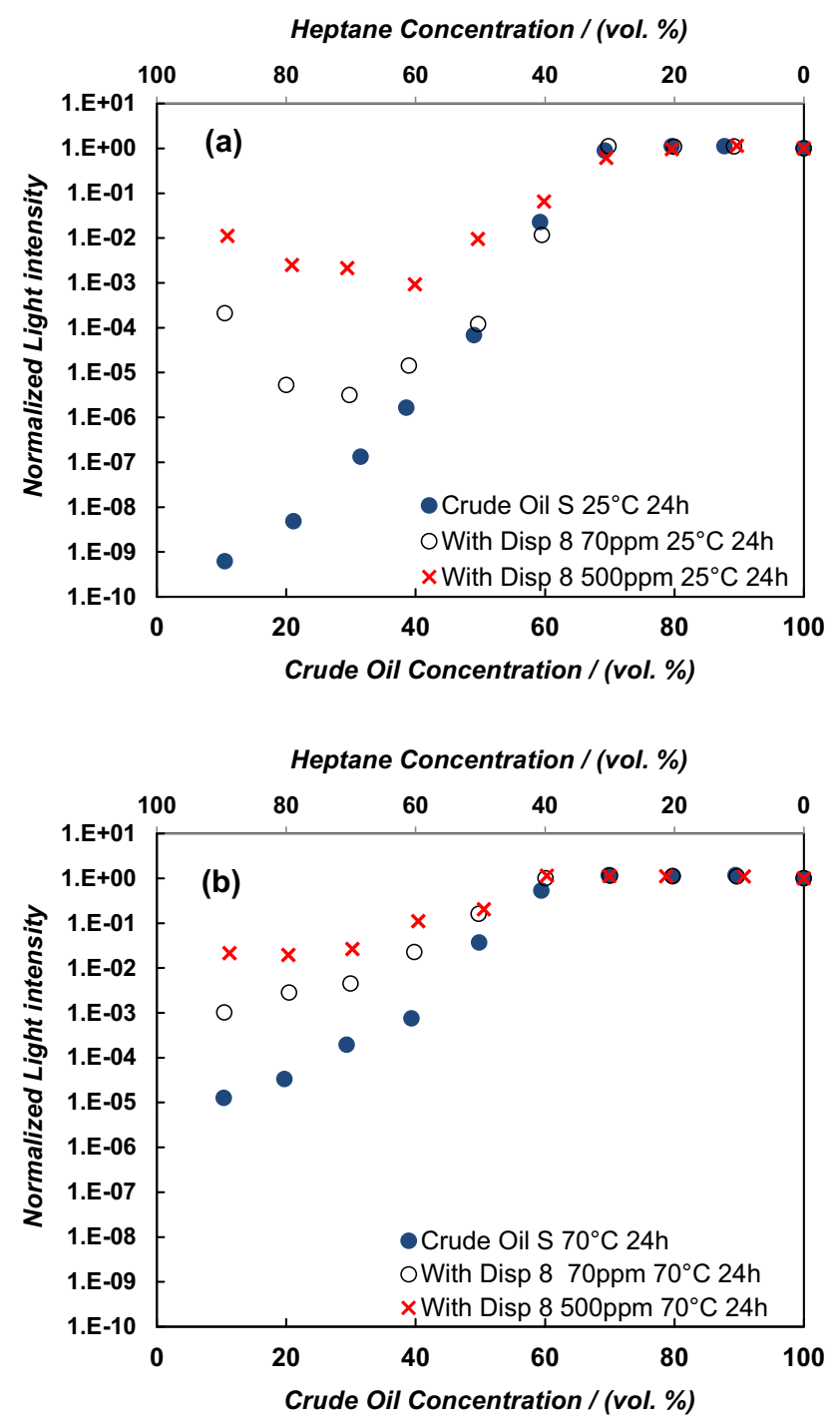

Fig. 8. Results of the direct method for the crude oil $S$ for (a) crude oil $S$ vs. dispersant 8 at 70 and $500 \mathrm{ppm}$ at $25^{\circ} \mathrm{C}$. (b) Crude oil S vs. dispersant 8 at 70 and $500 \mathrm{ppm}$ at $70^{\circ} \mathrm{C}$, after mixing with heptane for $24 \mathrm{~h}$. The ASD are $2.44 \%$ and $3.34 \%$ for crude oil S at $25^{\circ} \mathrm{C}$ and crude oil S at $70{ }^{\circ} \mathrm{C}, 4.68 \%$ and $6.20 \%$ for dispersant 8 at $70 \mathrm{ppm}$ and $500 \mathrm{ppm}$ at $25{ }^{\circ} \mathrm{C}$ and $5.98 \%$ and $1.89 \%$ for dispersant 8 at $70 \mathrm{ppm}$ and $500 \mathrm{ppm}$ at $70^{\circ} \mathrm{C}$ respectively.

$\mathrm{DPE}=100 *\left(1-\frac{A^{\text {Disp.Formulation }}}{A^{\text {Control }}}\right)$

$A=\int_{10}^{100}-\log _{10}($ Light intensity $) \mathrm{dC}_{\text {crude oil }}$ where $A^{\text {Control }}$ represents the area between the curve for the control and the base horizontal line and $A^{\text {Disp. }}$ represents the area between the curve for the treated system and the horizontal base line. The areas can be estimated using a numerical method, such as the trapezoidal rule. In this section the higher the DPE between dispersants evaluated at the same conditions, the more efficient the chemical additive as a dispersant. This index is a measurement on how well the chemical additive disperses asphaltenes, not how well it actually prevents asphaltene deposition. Table 5 presents the results for dispersants 8,9 and 15 at different concentrations, temperatures and aging time conditions. Because the dispersive performance for the crude oil S without dispersant is zero for all temperatures and aging time conditions it is not presented in Table 5.

Table 5 summarizes the dispersant efficiencies and restates the trends that have been already discussed in this document. As was previously discussed in Section 3.3.1 for the experiments performed at $70 \mathrm{ppm}, 25^{\circ} \mathrm{C}$ and $1 \mathrm{~h}$ aging time dispersant 8 was the most effective dispersant ( $\mathrm{DPE}=46.9 \%$ ), followed by dispersant 9 (DPE $=22.7 \%$ ) and dispersant 15 (DPE $=7.3 \%$ ).

At all aging times evaluated, i.e. $1 \mathrm{~h}, 24 \mathrm{~h}$ and 2 weeks, at $70 \mathrm{ppm}$ and $25^{\circ} \mathrm{C}$, the dispersant 8 performed better than dispersant 9 . After $24 \mathrm{~h}$ dispersant 8 ( DPE $=28.8 \%$ ) is more effective than dispersant 9 (DPE $=14.3 \%)$. Then, after two weeks dispersants 8 $(\mathrm{DPE}=15.8 \%)$ has a better efficiency than dispersant 9 $(\mathrm{DPE}=7.1 \%)$ as well.

When the dispersants concentration increased to $500 \mathrm{ppm}$, at $25^{\circ} \mathrm{C}$ and after $1 \mathrm{~h}$ aging time dispersant 9 had the best dispersive performance $(\mathrm{DPE}=54.1 \%)$, followed by dispersant $8(\mathrm{DPE}=51.5 \%)$ and dispersant 15 (DPE $=40.3 \%)$. In addition, as discussed in Section 3.3 .1 dispersant 8 at $70 \mathrm{ppm}, 25^{\circ} \mathrm{C}$ and after $1 \mathrm{~h}$ aging time had a dispersive performance ( $\mathrm{DPE}=46.9 \%$ ) very close to the dispersants at high concentration.

According to the previous discussion in Section 3.3.3 when the temperature is kept constant and the dispersant dosage was increased, a higher dispersive effect was observed. For dispersant 8 after $24 \mathrm{~h}$ aging time, the efficiency rose from $28.8 \%$ to $58 \%$ at $25{ }^{\circ} \mathrm{C}$ and from $42.2 \%$ to $62.1 \%$ at $70{ }^{\circ} \mathrm{C}$ when the dosage was increased from $70 \mathrm{ppm}$ to $500 \mathrm{ppm}$, respectively. At $70^{\circ} \mathrm{C}$, $70 \mathrm{ppm}$ and $24 \mathrm{~h}$ aging time dispersant 8 (42.2\%) once again was more effective than dispersant 9 (8.9\%).

The high reservoir temperatures can severely impact the dispersant performance efficiency. The chemical additives need to be designed to work at a wide range of temperature conditions. The dispersive performance efficiency should be calculated against a control that is held at the same temperature conditions.

Dispersant 8 showed the best performance at almost all conditions, followed by dispersant 9 and dispersant 15 . This trend only changed at $500 \mathrm{ppm}$ dosage, $25^{\circ} \mathrm{C}, 1 \mathrm{~h}$ aging time where dispersant 9 was slightly more efficient than dispersant 8 .

Table 5

Average (Avg) and Maximum (Max) dispersive performance efficiencies for Dispersants 8, 9 and 15 at different dosages, temperatures and aging time conditions.

\begin{tabular}{|c|c|c|c|c|c|c|c|c|c|}
\hline \multicolumn{10}{|c|}{ Dispersive performance efficiency (\%) } \\
\hline \multirow{3}{*}{ Sample } & \multirow{3}{*}{ Dosage (ppm) } & \multirow{2}{*}{\multicolumn{2}{|c|}{$\begin{array}{l}1 \mathrm{~h} \\
25^{\circ} \mathrm{C} \\
\end{array}$}} & \multicolumn{4}{|l|}{$24 \mathrm{~h}$} & \multirow{2}{*}{\multicolumn{2}{|c|}{$\begin{array}{l}2 \text { weeks } \\
25^{\circ} \mathrm{C}\end{array}$}} \\
\hline & & & & \multicolumn{2}{|l|}{$25^{\circ} \mathrm{C}$} & \multicolumn{2}{|c|}{$70^{\circ} \mathrm{C}$} & & \\
\hline & & Avg & Max & Avg & Max & Avg & Max & Avg & Max \\
\hline \multirow[t]{2}{*}{ Disp 8} & 70 & 46.9 & 58.3 & 28.8 & 66.0 & 42.2 & 43.0 & 15.8 & $50.0^{*}$ \\
\hline & 500 & 51.5 & 61.1 & 58.0 & 77.6 & 62.1 & 65.3 & NA & NA \\
\hline \multirow[t]{2}{*}{ Disp 9} & 70 & 22.7 & 41.9 & 14.3 & 49.3 & 8.9 & 10.4 & 7.1 & $30.8^{*}$ \\
\hline & 500 & 54.1 & 72.9 & - & - & - & - & - & - \\
\hline \multirow[t]{2}{*}{ Disp 15} & 70 & 7.3 & 15.4 & - & - & - & - & - & - \\
\hline & 500 & 40.3 & 68.8 & - & - & - & - & - & - \\
\hline
\end{tabular}


In the case of chemical additives that perform as asphaltene dispersants it is important to take into consideration as Vargas has reported [9], that reducing the particle size or in other words enhancing the dispersion effect might not necessarily help in preventing deposition.

\subsection{Indirect method}

In Section 3.3 it was noted that the addition of dispersants caused a slight shift on the onset of precipitation. To further investigate the underlying cause for this shift, the indirect method to detect the onset of asphaltene precipitation was employed. In Section 2.4, the features of the Indirect Method were briefly described. This method is much more sensitive to detect asphaltene precipitation. The indirect method was used to study the effect of dispersants 8 and 9 on asphaltene precipitation from the crude oil $\mathrm{S}$ diluted with n-heptane and aged for $24 \mathrm{~h}$ at $25^{\circ} \mathrm{C}$.

In order to be consistent the indirect method results were also plotted as a function of the normalized light intensity vs. crude oil vol.\% and heptane vol.\%. Fig. 9(a)-(c) presents the results. In this case the light intensity increase with respect to the horizontal line is the indication of the asphaltene precipitation. The increase in the light intensity is proportional to the amount of asphaltene precipitated from the oil. The more asphaltenes are removed upon precipitation the higher the intensity of the light.

Fig. 9(a) and (b) shows the absorbance values for dispersant 8 and 9 respectively at $70 \mathrm{ppm}, 25^{\circ} \mathrm{C}$ and $24 \mathrm{~h}$ aging time. In both cases with and without the addition of a dispersant the results are almost identical at the different concentrations of added nheptane. For dispersant 8, according to Fig. 9(c), even when the dosage is increased to $500 \mathrm{ppm}$ the results do not change significantly. Therefore, the presence of an asphaltene dispersant did neither affect the onset of precipitation nor the amount of precipitated asphaltenes.

After $24 \mathrm{~h}$ of aging time Fig. 9 shows a lower detection of asphaltene precipitation compared with the direct method results presented in Table 4 in Section 3.3.2 This is an indication of the greater sensitivity of the indirect method [31] that allows the detection of smaller particles.

The crude oil S was treated with 2000 ppm of dispersant 9, at $25^{\circ} \mathrm{C}$ for $24 \mathrm{~h}$ and it was evaluated using direct and indirect methods. Similarly to the observation from the HPHT test Fig. 10(a) shows the results for direct method and a shift in the detection of asphaltene precipitation for the treated system at ambient pressure. The detection of asphaltene precipitation increased from 22.4 to 28.8 n-heptane vol.\% when the concentration of inhibitor increased from 0 to $2000 \mathrm{ppm}$. This could be misinterpreted to mean, as in the SDS test, that the dispersant stabilizes the asphaltenes in crude oil, because there is a shift on the detection of asphaltene precipitation towards a greater precipitant amount. However, Fig. 10(b) presents the indirect method results and there was neither a change on the detection of asphaltene precipitation nor the amount of asphaltene precipitate observed.

To reconcile these results, one should look at the limitation of the techniques used. Once the asphaltenes start precipitating, the process leads to the formation of small particles in the order of $100-400 \mathrm{~nm}[31,38]$ that can further aggregate at later stages. The detection limit of the direct method is close to $1 \mu \mathrm{m}$ [31]. Thus, although the precipitated particles may already be present in the solution the onset of precipitation via direct method can only be detected when the asphaltene aggregates have grown to exceed the minimum detection limit. Since the aggregation rate is slowed down by the action of the dispersant, the particle size remains undetectable for a longer time or while larger amounts of
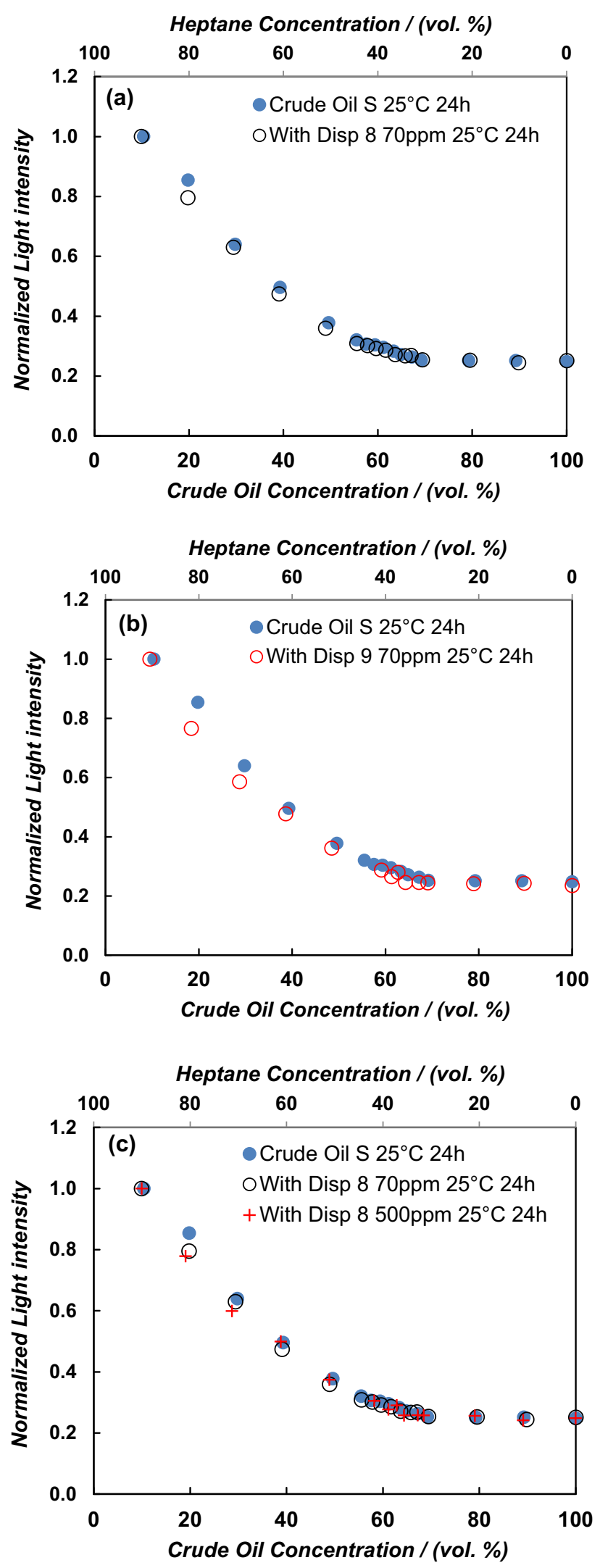

Fig. 9. Indirect method results reported as normalized light intensity vs. crude oil vol.\% and heptane vol.\% concentrations for crude oil S with (a) dispersant 8 at 70 ppm, (b) dispersant 9 at 70 ppm, (c) dispersant 8 at 70 ppm and 500 ppm, after mixing with heptane and aging for $24 \mathrm{~h}$ at $25{ }^{\circ} \mathrm{C}$. The ASD is $2.15 \%, 1.51 \%, 1.4 \%$ and $1.0 \%$ for the crude oil S, dispersant 8 and dispersant 9 at $70 \mathrm{ppm}$, dispersant 8 at 500 ppm respectively. 

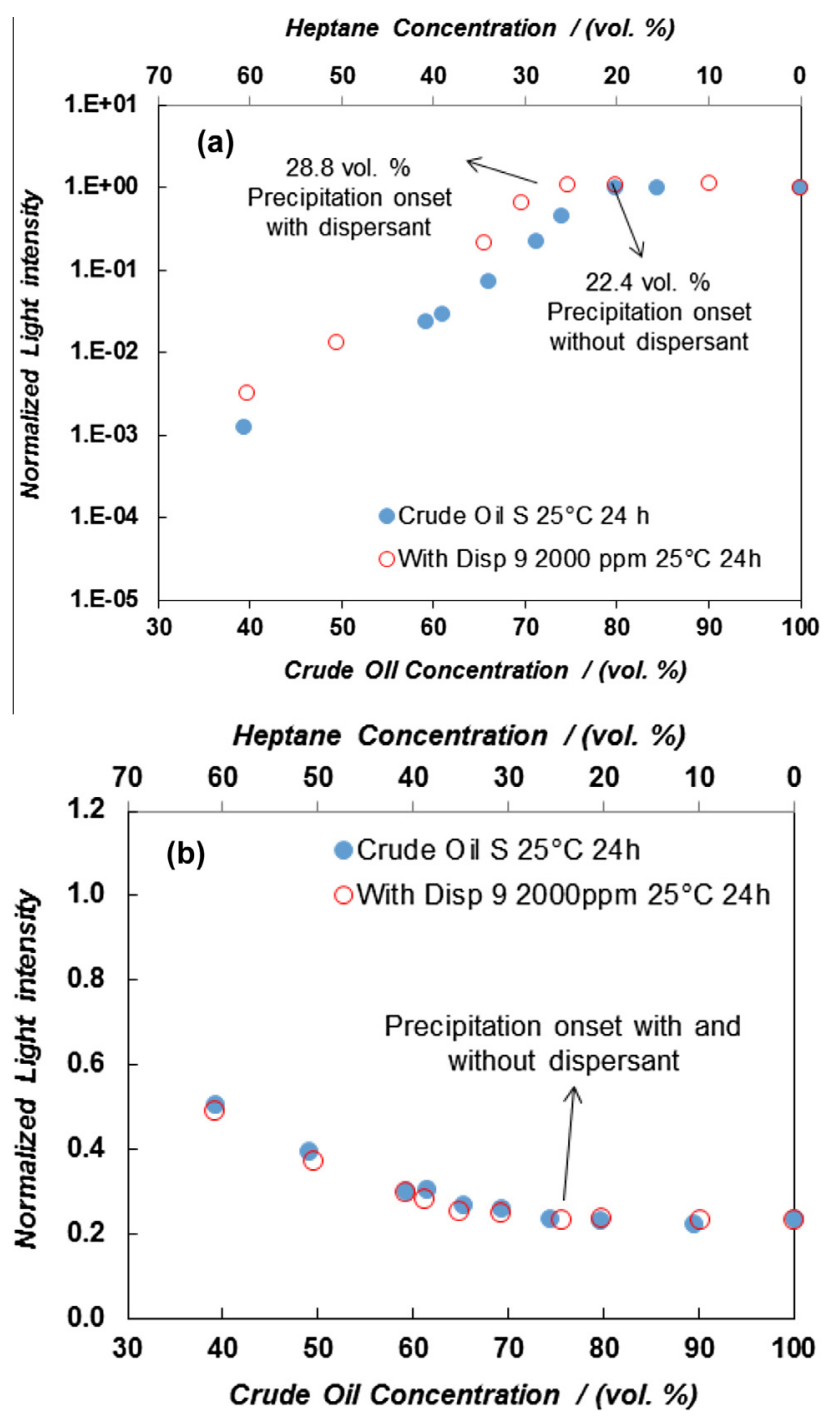

Fig. 10. Results for crude oil S with a 2000-ppm dosage of dispersant 9, after mixing with heptane and aging for $24 \mathrm{~h}$ at $25^{\circ} \mathrm{C}$, (a) direct method. The ASD are $3.17 \%$ and $3.27 \%$ for the crude oil S and dispersant 9 respectively. (b) Indirect method. The ASD are $1.73 \%$ and $2.32 \%$ for the crude oil S and dispersant 9 respectively.

asphaltene precipitant are added, which gives the false impression of increased stability. Because the indirect method is much more sensitive, and particles as small as $100 \mathrm{~nm}$ can be detected, it is possible to differentiate the precipitation and aggregation steps. Consequently, the dispersants do not seem to affect the onset of asphaltene precipitation, only the rate of subsequent aggregation.

\section{Conclusions}

Asphaltene precipitation, aggregation and deposition are part of a complex multi-step mechanism that it is still under active investigation. In this work, precipitation of asphaltene is defined as the separation of a distinct asphaltene rich-phase from an asphaltene lean-phase. Asphaltene particles, as small as a few hundred nanometers, begin to form at the onset of asphaltene precipitation. These small particles can then, either further aggregate or get dispersed and eventually deposit on an available surface. According to the results presented in this work commercial asphaltene dispersants interact with the asphaltenes in the oil to slow down the asphaltene aggregation rate. Conventional techniques, based on NIR transmittance or optical microscopy, are unable to detect the onset of asphaltene precipitation, due to their insufficient sensitivity and, consequently, they may provide misleading results that suggest that the addition of the inhibitor causes a shift on the thermodynamic stability of the asphaltenes. Actually, the precipitation of asphaltenes occurs at the same ratio of oil/n-heptane for treated and untreated systems, according to the results obtained with the indirect method, which is about one order of magnitude more sensitive than conventional techniques used to determine the onset of asphaltene precipitation. The experimental evidence shows that the asphaltene dispersants neither shift the actual onset of asphaltene precipitation nor reduce the amount of asphaltene precipitated. Because the precipitation of asphaltenes is not affected by the dispersant, the dispersion efficiency should not be evaluated considering the shift in the detection of asphaltene precipitation as the evaluation parameter.

Also, the analysis of the performance of the chemicals used to disperse asphaltenes is usually done through the Asphaltene Dispersion Test (ADT), which analyzes dead oil samples at ambient conditions. Also, the ADT requires a high dilution ratio with n-heptane. The results presented in this work provide experimental evidence against the reliability of the ADT. For example, the experiments performed at 25 and $70{ }^{\circ} \mathrm{C}$ reveal that the maximum efficiency of the dispersants is reduced for increasing temperatures. This can be due to the effect of the temperature on the ability of the chemical to disperse asphaltenes or simply because in general the solubility of asphaltenes increases with increasing temperature, and therefore the addition of a dispersant provides a reduced benefit for systems at higher temperature. In either case the ranking of chemicals based on their performance can be greatly affected by the temperature at which the experiments are conducted. The same is true for the effect of the concentration of the blends analyzed. In the ADT, the oil, with or without dispersant, is mixed with n-heptane to produce blends than contain up to $97 \%$ of $n$-heptane. The performance of the chemical was also found to be highly dependent on the ratio of n-heptane to oil. Thus, a successful method to analyze and rank the performance of asphaltene dispersants at ambient pressure should take, as a minimum, the effect of temperature and composition into consideration.

The dispersive performance was introduced as a new index to evaluate the dispersants formulations efficiency. It allows ranking and comparing different dispersant formulations under the same conditions. The results found through this index agree with theobservations from the graphical analysis. As expected, the dispersive performance of all the chemicals increased with the increasing concentration of the dispersant. At $500 \mathrm{ppm}$ the three commercial inhibitors that were tested behaved similarly, and only one of them held an acceptable performance at concentrations as low as $70 \mathrm{ppm}$. Thus, it is recommended to investigate the performance of chemicals at the lower concentrations, such as 50-100 ppm, which is a more desirable formulation from an economic perspective.

Because the ultimate goal of using these chemicals is to reduce the amount of asphaltene deposits obtained in production tubing, it is greatly desirable to study the performance of these chemicals to reduce actual deposition. This is the subject of a current research project, whose results will be presented in a separate research article.

\section{Acknowledgments}

This work was undertaken with the generous support of $\mathrm{Abu}$ Dhabi National Oil Company (ADNOC) Research and Development Oil Subcommittee. 


\section{Appendix A. Supplementary material}

Supplementary data associated with this article can be found, in the online version, at http://dx.doi.org/10.1016/j.fuel.2016.03.056.

\section{References}

[1] Kabir CS, Jamaluddin AKM, et al. Asphaltene characterization and mitigation in south Kuwait's Marrat reservoir. SPE Prod Facil 2002;17:251-8.

[2] León O, Rogel E, Espidel J, Torres G. Asphaltenes: structural characterization, self-association, and stability behavior. Energy Fuels 2000;14:6-10. http://dx. doi.org/10.1021/ef9901037.

[3] Calemma V, Iwanski P, Nali M, Scotti R, Montanari L. Structural characterization of asphaltenes of different origins. Energy Fuels 1995;9:225-30. http://dx.doi.org/10.1021/ef00050a004.

[4] Ancheyta J, Centeno G, Trejo F, Marroquín G, García JA, Tenorio E, et al. Extraction and characterization of asphaltenes from different crude oils and solvents. Energy Fuels 2002;16:1121-7. http://dx.doi.org/10.1021/ef010300h.

[5] Wu J, Prausnitz JM, Firoozabadi A. Molecular-thermodynamic framework for asphaltene-oil equilibria. AIChE J 1998;44:1188-99. http://dx.doi.org/ 10.1002/aic.690440516.

[6] Goual L, Sedghi M, Mostowfi F, McFarlane R, Pomerantz AE, Saraji S, et al. Cluster of asphaltene nanoaggregates by DC conductivity and centrifugation. Energy Fuels 2014. http://dx.doi.org/10.1021/ef5010682.

[7] Mullins OC. The modified yen model †. Energy Fuels 2010;24:2179-207. http:// dx.doi.org/10.1021/ef900975e.

[8] Sedghi M, Goual L, Welch W, Kubelka J. Effect of asphaltene structure on association and aggregation using molecular dynamics. J Phys Chem B 2013;117:5765-76. http://dx.doi.org/10.1021/ip401584u.

[9] Vargas FM, Creek JL, Chapman WG. On the development of an asphaltene deposition simulator. Energy Fuels 2010;24:2294-9. http://dx.doi.org/ 10.1021/ef900951n.

[10] Rocha Junior LC, Ferreira MS, da Silva Ramos AC. Inhibition of asphaltene precipitation in Brazilian crude oils using new oil soluble amphiphiles. J Pet Sci Eng 2006;51:26-36. http://dx.doi.org/10.1016/i.petrol.2005.11.006.

[11] Vargas FM. Modeling of asphaltene precipitation and arterial deposition. Rice University; 2009.

[12] Juyal P, Ho V, Yen A, Allenson SJ. Reversibility of asphaltene flocculation with chemicals. Energy Fuels 2012;26:2631-40. http://dx.doi.org/10.1021/ ef201389e.

[13] Lighfford S, Pitoni E, Armesi F, Mauri L. Development and field use of a novel solvent/water emulsion for the removal of asphaltene deposits in fractured carbonate formations. Spe Prod Oper 2008;23:301-11. http://dx.doi.org/ 10.2118/101022-PA.

[14] Rogel E. Effect of inhibitors on asphaltene aggregation: a theoretical framework $\dagger$. Energy Fuels 2010;25:472-81.

[15] Ibrahim HH, Idem RO. Interrelationships between asphaltene precipitation inhibitor effectiveness, asphaltenes characteristics, and precipitation behavior during n-heptane (light paraffin hydrocarbon)-induced asphaltene precipitation. Energy Fuels 2004;18:1038-48. http://dx.doi.org/10.1021/ ef0340460.

[16] Trbovich MG, King GE, et al. Asphaltene deposit removal: long-lasting treatment with a co-solvent. In: SPE Int. Symp. Oilfield Chem. Society of Petroleum Engineers; 1991.

[17] Ghloum EF, Al-Qahtani M, Al-Rashid A. Effect of inhibitors on asphaltene precipitation for Marrat Kuwaiti reservoirs. J Pet Sci Eng 2010;70:99-106. http://dx.doi.org/10.1016/i.petrol.2009.10.003.

[18] González Fabio A. Personal communication. Asphaltene deposition economic impact. Reservoir performance global community of practice lead BP; 2015.

[19] Abdallah Dalia S. Personal communication. Abu Dhabi, UAE: Abu Dhabi Company for Onshore Petroleum Operations Ltd.; 2015.

[20] González G, Middea A. Peptization of asphaltene by various oil soluble amphiphiles. Colloids Surf 1991;52:207-17. http://dx.doi.org/10.1016/0166$\underline{6622(91) 80015-G}$.
[21] Wang J, Li C, Zhang L, Que G, Li Z. The properties of asphaltenes and their interaction with amphiphiles. Energy Fuels 2009;23:3625-31. http://dx.doi. org/10.1021/ef801148y.

[22] Yen A, Yin YR, Asomaning S. Evaluating asphaltene inhibitors: laboratory tests and field studies. Society of Petroleum Engineers; 2001. http://dx.doi.org/ 10.2118/65376-MS.

[23] Barcenas M, Orea P, Buenrostro-González E, Zamudio-Rivera LS, Duda Y. Study of medium effect on asphaltene agglomeration inhibitor efficiency. Energy Fuels 2008;22:1917-22. http://dx.doi.org/10.1021/ef700773m.

[24] Smith DF, Klein GC, Yen AT, Squicciarini MP, Rodgers RP, Marshall AG. Crude oil polar chemical composition derived from FT-ICR mass spectrometry accounts for asphaltene inhibitor specificity. Energy Fuels 2008;22:3112-7. http://dx.doi.org/10.1021/ef800036a.

[25] Barcenas M, Orea P. Molar-mass distributions of asphaltenes in the presence of inhibitors: experimental and computer calculations. Energy Fuels 2011;25:2100-8. http://dx.doi.org/10.1021/ef200108t.

[26] Chang C-L, Fogler HS. Stabilization of asphaltenes in aliphatic solvents using alkylbenzene-derived amphiphiles. 1. Effect of the chemical structure of amphiphiles on asphaltene stabilization. Langmuir 1994;10:1749-57. http:// dx.doi.org/10.1021/la00018a022.

[27] Chang C-L, Fogler HS. Stabilization of asphaltenes in aliphatic solvents using alkylbenzene-derived amphiphiles. 2. Study of the asphaltene-amphiphile interactions and structures using Fourier transform infrared spectroscopy and small-angle X-ray scattering techniques. Langmuir 1994;10:1758-66. http:/ dx.doi.org/10.1021/la00018a023.

[28] Kraiwattanawong K, Fogler HS, Gharfeh SG, Singh P, Thomason WH, Chavade $\mathrm{S}$. Effect of asphaltene dispersants on aggregate size distribution and growth. Energy Fuels 2009;23:1575-82. http://dx.doi.org/10.1021/ef800706c.

[29] Al-Sahhaf TA, Fahim MA, Elkilani AS. Retardation of asphaltene precipitation by addition of toluene, resins, deasphalted oil and surfactants. Fluid Phase Equilib 2002;194-197:1045-57. http://dx.doi.org/10.1016/S0378-3812(01) 00702-6.

[30] Oh K, Deo MD. Effect of organic additives on the onset of asphaltene precipitation. Energy Fuels 2002;16:694-9. http://dx.doi.org/10.1021 ef010223q.

[31] Tavakkoli M, Grimes MR, Liu X, Garcia CK, Correa SC, Cox QJ, et al. Indirect method: a novel technique for experimental determination of asphaltene precipitation. Energy Fuels 2015;29:2890-900. http://dx.doi.org/10.1021/ ef502188u.

[32] Goual L, Sedghi M, Wang X, Zhu Z. Asphaltene aggregation and impact of alkylphenols. Langmuir 2014;30:5394-403. http://dx.doi.org/10.1021 la500615k.

[33] Mohammadi M, Akbari M, Fakhroueian Z, Bahramian A, Azin R, Arya S. Inhibition of asphaltene precipitation by $\mathrm{TiO}_{2}, \mathrm{SiO}_{2}$, and $\mathrm{ZrO}_{2}$ nanofluids. Energy Fuels 2011;25:3150-6. http://dx.doi.org/10.1021/ef2001635.

[34] Carnahan NF, Salager J-L, Antón R, Dávila A. Properties of resins extracted from boscan crude oil and their effect on the stability of asphaltenes in boscan and hamaca crude oils. Energy Fuels 1999;13:309-14. http://dx.doi.org/10.1021 ef980218v.

[35] Fuhr BJ, Klein LL, Reichert C. Measurement of asphaltene flocculation in bitumen solutions. J Can Pet Technol 1986:25. http://dx.doi.org/10.2118/8605-02.

[36] Tavakkoli M, Panuganti SR, Taghikhani V, Pishvaie MR, Chapman WG. Asphaltene deposition in different depositing environments: Part 2. Real oil. Energy Fuels 2014;28:3594-603. http://dx.doi.org/10.1021/ef401868d.

[37] Vargas FM, Gonzalez DL, Hirasaki GJ, Chapman WG. Modeling asphaltene phase behavior in crude oil systems using the perturbed chain form of the statistical associating fluid theory (PC-SAFT) equation of state + . Energy Fuels 2009;23:1140-6. http://dx.doi.org/10.1021/ef8006678.

[38] Vargas F, Garcia-Bermudes M, Boggara M, Punnapala S, Abutaqiya MIL, Mathew NT, et al. On the development of an enhanced method to predict asphaltene precipitation. Houston, TX: Offshore Technology Conference; 2014 http://dx.doi.org/10.4043/25294-MS. 